\title{
Bacterial Adhesion to Grade 4 and Grade 5 Turned and Mildly Acid-Etched Titanium Implant Surfaces: An In Vitro and Ex Vivo Study
}

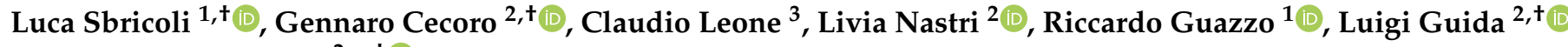 \\ and Marco Annunziata $2, *,+$ (iD \\ 1 Department of Neurosciences, School of Dentistry, University of Padova, Via Giustiniani 2, \\ 35100 Padova, Italy; luca.sbricoli@unipd.it (L.S.); riccardo.guazzo@unipd.it (R.G.) \\ 2 Multidisciplinary Department of Medical-Surgical and Dental Specialties, University of Campania “Luigi \\ Vanvitelli", Via L. De Crecchio 6, 80138 Naples, Italy; gennarocecoro@gmail.com (G.C.); \\ livia.nastri@unicampania.it (L.N.); luigi.guida@unicampania.it (L.G.) \\ 3 Department of Engineering, University of Campania "Luigi Vanvitelli", via Roma 29, \\ 81031 Aversa (Caserta), Italy; claudio.leone@unicampania.it \\ * Correspondence: marco.annunziata@unicampania.it; Tel.: +39-0815665515 \\ + L.S. and G.C. share the first authorship; L.G. and M.A. share the last authorship.
}

\section{check for}

updates

Citation: Sbricoli, L.; Cecoro, G.; Leone, C.; Nastri, L.; Guazzo, R.; Guida, L.; Annunziata, M. Bacterial Adhesion to Grade 4 and Grade 5 Turned and Mildly Acid-Etched Titanium Implant Surfaces: An In Vitro and Ex Vivo Study. Appl. Sci. 2021, 11, 7185. https://doi.org/ 10.3390/app11167185

Academic Editor:

Tommaso Lombardi

Received: 11 June 2021

Accepted: 2 August 2021

Published: 4 August 2021

Publisher's Note: MDPI stays neutral with regard to jurisdictional claims in published maps and institutional affiliations.

Copyright: (c) 2021 by the authors. Licensee MDPI, Basel, Switzerland. This article is an open access article distributed under the terms and conditions of the Creative Commons Attribution (CC BY) license (https:/ / creativecommons.org/licenses/by/ $4.0 /)$.
Abstract: Aim of the present study was to investigate the bacterial adhesion to titanium (Ti) implant surfaces, different for composition and topographic features. Grade 4 and 5 turned (T-4, T-5,) and mildly acid-etched (MA-4, MA-5) Ti $6 \times 1 \mathrm{~mm}$ disks were topographically analyzed by scanning electronic microscopy and 3D profilometry. Bacterial cultures (Streptococcus sanguinis) were in vitro seeded and, after two and six hours, adherent bacteria were quantified by colony-forming unit (CFU) counting. Ti samples were also exposed to the oral environment of six periodontally healthy volunteers and, after $12 \mathrm{~h}$, the formed biofilm was evaluated by CFU counting. Inter-group differences were tested by the Mann-Whitney U-Test $(\alpha=0.05)$. MA surfaces were significantly rougher than T ones, whereas no difference between grade 4 and grade 5 disks was detected. Significantly higher in vitro bacterial adhesion for MA than T disks was shown at two and six hours. Significantly higher values of CFU counting for MA than T surfaces and for grade 5 than grade 4 disks were found at the 12 h-ex vivo test. Bacterial adhesion showed to be sensitive to both Ti surface topography and composition, with possible implications on peri-implant tissue health maintenance.

Keywords: dental implants; bacterial adhesion; surface properties

\section{Introduction}

The use of titanium (Ti) dental implants is, nowadays, the main rehabilitative option in case of edentulism, with high clinical performance demonstrated in the long-term [1].

The osseointegration process has been demonstrated to be strongly affected by surface properties of Ti implants [2], and it has been suggested that such properties may be crucially associated with the maintenance of osseointegration over time [3].

The modification of topographic properties of Ti surfaces, by industrial treatments aimed to increase surface roughness, represents a consolidated strategy to promote implant osseointegration [4,5].

On the other hand, surface properties, particularly surface roughness, have been reported to promote microbial adhesion [6,7] and, possibly, the onset or the progression of peri-implant diseases [3]. An implant surface harboring the biofilm may lead to an inflammatory/infectious peri-implant lesion [8], although the link between implant surface and peri-implant disease is still far from being clearly defined. Definitive data, indeed, are lacking and, at the present time, implant roughness is not mentioned as a risk indicator for peri-implant diseases [8-10]. 
Furthermore, chemical surface properties and Ti composition also may affect the biological behavior and, besides, chemical, topographic and biological features have been demonstrated to be linked to each other [4].

The main Ti alloy commonly used is the so-called commercially pure Ti, cpTi [11]. It is available in four grades numbered 1 to 4 , according to the purity and the processing oxygen content [12], with the grade $4 \mathrm{cp}-\mathrm{Ti}$, having the highest oxygen content (around $0.4 \%$ ) and the best overall mechanical strength, which is most widely used for dental implants [11,12]. Another Ti alloy used for manufacturing of surgical and, especially, prosthetic implant components is the Ti-6Al-4V, also called grade $5 \mathrm{Ti}$, that shows superior strength, lower Young's modulus and acceptable biological properties [13].

Little data about the effect of Ti composition on bacterial adhesion are available to date, with contrasting results. Barão et al. [14], for instance, demonstrated significantly more Porphyromonas gingivalis attached on cp-Ti disks than on Ti-6Al-4V alloy disks after six hours of anaerobic incubation. Differently, Di Giulio et al. [15] showed that grade 4 Ti surfaces were significantly less attractant for the $P$. gingivalis than grade 5 Ti machined and lasertreated surfaces, whereas they found no detectable difference on sandblasted specimens.

Such studies, however, were limited by the use of an exclusively in vitro mono-species experimental model, whereas the effect of both Ti composition and topography on the biofilm formation occurring in conditions closer to those of the oral cavity are lacking to date.

Thus, the aim of the present study is to evaluate the interaction of oral bacteria with differently composed and treated Ti implant surfaces, and to verify how surface chemical and topographic properties may affect both bacterial adhesion in vitro, and biofilm formation ex vivo.

\section{Materials and Methods}

\subsection{Products and Reagents}

All cell culture biologicals were purchased from Gibco BRL (Grand Island, NY, USA), and all chemicals were from Sigma Chemical Co. (St. Louis, MO, USA) when not otherwise specified.

\subsection{Specimen Preparation}

Four different Ti implant surfaces were analyzed, different for surface treatment and chemical composition (Ti purity): grade 4 turned (T-4), grade 5 turned (T-5), grade 4 mildly acid-etched (MA-4), and grade 5 mildly acid-etched (MA-5) Ti surfaces. Ti discs (6 mm wide and $1 \mathrm{~mm}$ thick) were provided by a commercial firm (Leader Italia s.r.l, Milano, Italia).

MA samples were obtained through "biological organic acid treatment" (B.O.A.T., Leader Italia s.r.l, Milano, Italia) including the following steps: sonication for $5 \mathrm{~min}$ in distilled water at $25^{\circ} \mathrm{C}$, immersion in $\mathrm{NaOH}(20 \mathrm{~g} / \mathrm{L})$ and hydrogen peroxide $(20 \mathrm{~g} / \mathrm{L})$ at $80{ }^{\circ} \mathrm{C}$ for $30 \mathrm{~min}$, further sonication for $5 \mathrm{~min}$ in distilled water, immersion in a mixture of $50 \%$ oxalic acid and $50 \%$ maleic acid at $80^{\circ} \mathrm{C}$ for $45 \mathrm{~min}$, sonication for $5 \mathrm{~min}$ in distilled water, immersion for $30 \mathrm{~min}$ in a solution of $65 \%$ nitric acid and distilled water with a volumetric range of 1 to 1 at $100{ }^{\circ} \mathrm{C}$, and washing in distilled water.

All Ti disks were sterilized in gamma radiation and singularly packed in sealed envelopes until use.

\subsection{Surface Characterization}

Qualitative and quantitative measurements of Ti surfaces were made by a 3D Surface Profiling System. In parallel, scanning electron microscopy (SEM) was used to visualize the topographic features of implant samples on a larger spatial range, and was coupled with an energy dispersive spectroscopy (EDS) analysis for the determination of surface chemical composition. 


\subsubsection{D Surface Profiling}

Surface topography was evaluated by a 3D Surface Profiling System (Talysurf CLI 2000 from Taylor Hobson), equipped with an inductive gauge $2 \mu \mathrm{m}$ radius diamond stylus. Further, $0.5 \times 0.5 \mathrm{~mm}^{2}$ surfaces were acquired, adopting the following setting: axis resolution (data spacing) $0.5 \mu \mathrm{m}$, lateral resolution $0.5 \mu \mathrm{m}$, and vertical resolution $2 \mathrm{~nm}$. A surface analysis software (Taly Map Universal, release 3.1) was used to elaborate the acquired profiles and measure roughness by multiple parameters, with the possibility for applying different filters.

The following 3D roughness parameters were analyzed: the absolute deviation of the surface (Sa, i.e., the arithmetic mean of the deviations from the mean, also known as average roughness), the density of summits of the surface (Sds, i.e., the number of peaks for unit area expressed in peaks $/ \mathrm{mm}^{2}$ ), and the developed surface area ratio ( $\mathrm{Sdr}$, i.e., the ratio between the area of the real developed surface and the area of the projected surface); the latter is an expression of the surface complexity.

Before the surface analysis, to separate the roughness profile from the primary profile, an $80 \mu \mathrm{m}$ Gauss' filters was applied on the acquired profiles. No less than four surfaces were analyzed for each surface type.

\subsubsection{SEM-EDS Analysis}

One sample for each surface was analyzed by Scanning Electron Microscopy / Energy Dispersive X-ray Spectrometry (SEM/EDS) carried out by an SEM (Quanta 200, FEI Europe Company, The Netherlands) at $25 \mathrm{kV}$ coupled with an EDS analyzer (TEAM ${ }^{\mathrm{TM}}$ EDS, EDAX Inc., Mahwah, NJ, USA). Samples were not metalized, to keep surface properties undamaged. Measurements were performed in quadruplicate of each examined particle and area of the surface.

\subsection{In Vitro Bacterial Adhesion}

\subsubsection{Bacterial Culture}

The bacteria cell line used in this study was Streptococcus sanguinis from the American Type Culture Collection (ATCC 10556). The dry pellet was rehydrated in $6 \mathrm{~mL}$ of Brain Heart Infusion (BHI; BD Bioscience, Franklin Lakes, NJ, USA) broth. The bacteria solution was agitated under standard cell conditions for $24 \mathrm{~h}$. The second passage of bacteria was diluted at a ratio of 1:200 into fresh $\mathrm{BHI}$ and incubated until it reached the stationary phase. The next passage was then frozen in one part $\mathrm{BHI}$ and one part glycerol and stored at $-18{ }^{\circ} \mathrm{C}$. All experiments were conducted from this frozen stock.

A stock of bacteria from the frozen stock was inoculated in a centrifuge tube with $3 \mathrm{~mL}$ of fresh BHI one day before bacterial seeding for the adhesion experiments. The cellular suspension was then adjusted to an optical density of about 1 at $495 \mathrm{~nm}$, diluted 1:40 in the same broth, and incubated for $7 \mathrm{~h}$, corresponding to exponential growth phase $/ \mathrm{mid}$ log.

\subsubsection{ESEM Analysis}

Ti disks were placed on the bottom of 24-well culture plate and bacteria were seeded on at a density of $1 \times 10^{7}$ bacteria $/ \mathrm{mL}$ (estimated by the McFarland scale). The optimal plating density to maintain enough residual space for surface colonization was detected by preliminary adhesion experiments.

After 2 and $6 \mathrm{~h}$ under standard conditions ( $5 \% \mathrm{CO}_{2} / 95 \%$ humidified air at $\left.37^{\circ} \mathrm{C}\right)$, loosely bound cells were removed by rinsing twice with phosphate-buffered solution (PBS). Adhering bacteria were observed using the scanning electron microscopy in environmental mode (ESEM), with a Peltier Stage and gaseous secondary electron detector. Chamber parameters were settled to $5{ }^{\circ} \mathrm{C}$ temperature and 3.60 Torr pressure, at $15 \mathrm{kV}$, with samples retaining $60 \%$ of relative humidity. 


\subsubsection{Determination of Bacterial Adhesion}

Bacterial growth from 2 day cultures was harvested and the optical density (OD) in each tube was adjusted to $1 \times 10^{7}$ bacteria $/ \mathrm{mL}$ by diluting the BHI bacteria cultures (Sigma Aldrich, St. Louis, MO, USA). Ti disks were placed individually in 12 well plates and a suspension of reference strain was added in a total volume of $2 \mathrm{~mL}$. Plates were incubated for 2 and $6 \mathrm{~h}$ at $37^{\circ} \mathrm{C}$ and $5 \% \mathrm{CO}_{2} / 95 \%$ humidified air. After incubation, each sample was washed twice with PBS to remove loosely bound cells, then sonicated 5 times for $10 \mathrm{~s}$ to detach adherent cells. Preliminary experiments were conducted to detect the sonication protocol not associated with a loss of viability of the cells. Collected bacteria were thoroughly Vortex-treated, and their number was measured by direct counting of colony-forming units (CFU) grown in agar plates incubated for $24 \mathrm{~h}$ at $37{ }^{\circ} \mathrm{C}$ and $5 \%$ $\mathrm{CO}_{2} / 95 \%$ humidified air, after serial dilutions.

Each experiment was carried out three times in triplicate.

\subsection{Ex Vivo Bacterial Adhesion}

Six subjects in excellent systemic health and periodontal conditions were selected to participate in the present study ( 3 males, 3 females; mean age 29 years; all non-smokers). Volunteers were not expected to have used systemic antibiotics for 30 days before the start of the study. Oral examination was carried out by an experienced dentist. All volunteers had physiological salivary flow rates $(1-1.5 \mathrm{~mL} / \mathrm{min}$ ) and excellent oral hygiene (plaque index and sulcus bleeding index close to zero). Informed written consent was given by the subjects and the protocol was approved by the Ethics Committee of the University of Campania "Luigi Vanvitelli" (\#602, 22 April 2015).

The Ti specimens were fixed to individual removable acrylic templates previously disinfected in $0.2 \%$ chlorhexidine for $20 \mathrm{~min}$ and then washed in distilled water. Four Ti specimens corresponding to different surface properties were positioned bilaterally in the buccal area of premolars and molars, so that biofilm growth was not disturbed by tongue movements. Food or beverage consumption and oral hygiene were forbidden during the carriage.

The templates were worn for $12 \mathrm{~h}$, and then the plaque-covered specimens were removed from the templates and immediately transferred to well plates, washed twice in PBS to remove non-adhering cells and then sonicated. The number of adherent bacteria was measured by direct CFU counting as previously described.

\subsection{Statistical Analysis}

Measured data were expressed as medians with interquartile ranges (25-75th percentile) and minimum/maximum values. The differences between groups were tested by the Mann-Whitney U test with the alpha-level set at $5 \%$.

\section{Results}

\subsection{Surface Characterization of Ti Samples}

\subsubsection{SEM/EDS Analysis}

At the SEM analysis specific and clearly distinguishable surface characteristics for turned samples, T-4 and T-5, and for mildly acid-etched samples, MA-4 and MA-5 (Figure 1) accordingly with the surface treatment applied, could be observed.

Turned surfaces showed an anisotropic texture with concentric microgrooves due to the producing process. MA samples did not show this pattern, probably because the adjunctive etching treatment reduced the entity of the microgrooves. The MA surface was characterized by repeated concavities, pits and ridges of homogeneous dimensions produced by the organic acids.

A similar composition for all the tested surfaces was measured by EDS analysis, with a high peak of Ti and adjunctive lower peaks of $\mathrm{Al}$ and $\mathrm{V}$ proper of the grade $5 \mathrm{Ti}$ alloy for T- 5 and MA- 5 samples, whereas in none of the surfaces tested were detected organic or inorganic contaminants (Figure 2). 

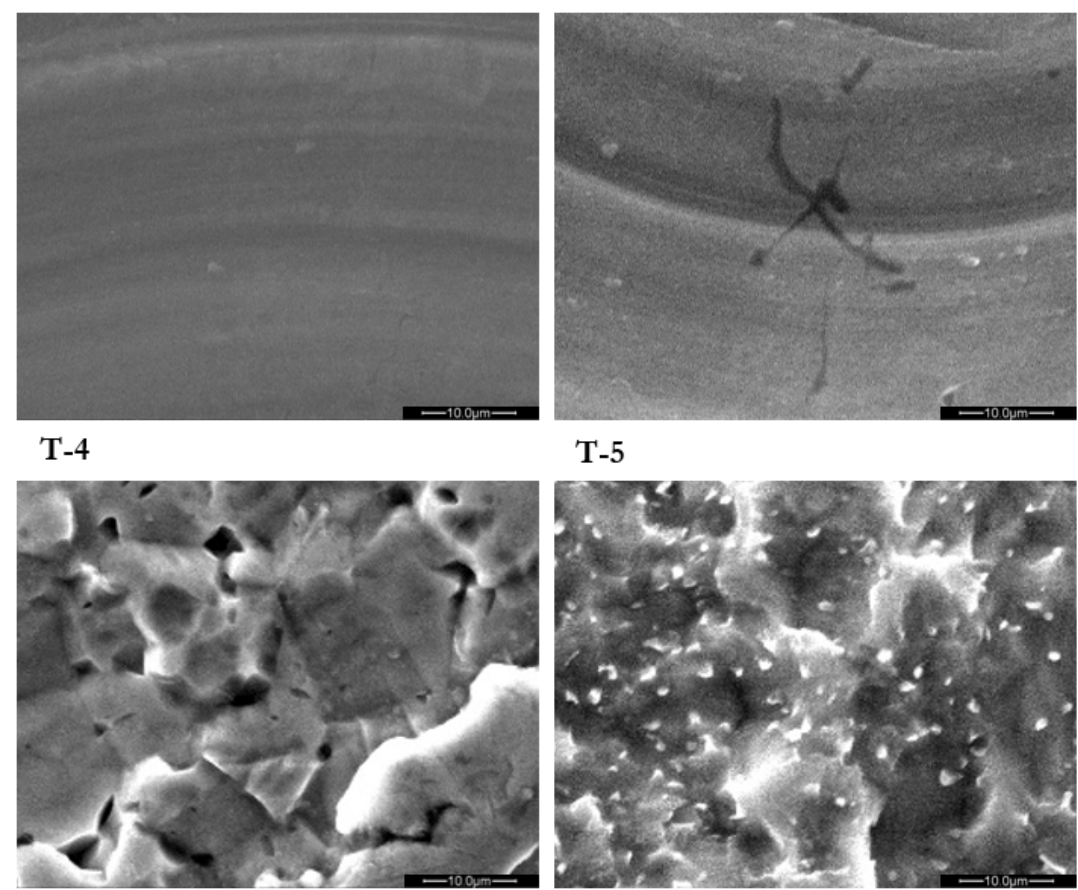

T-5

MA-4

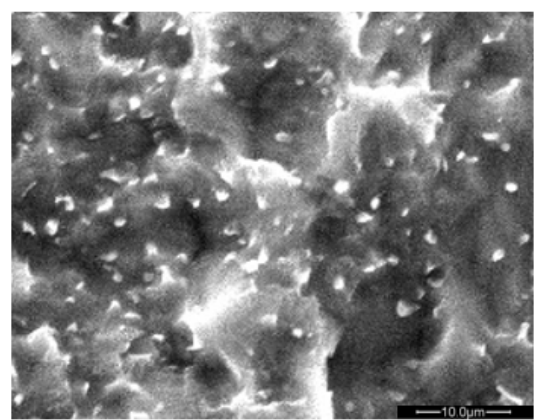

MA-5

Figure 1. Turned grade 4 (T-4), turned grade 5 (T-5), mildly acid-etched grade 4 (MA-4), and mildly acid-etched grade 5 (MA-5) Ti surfaces analyzed by scanning electron microscopy (SEM). Magnification $2500 \times$.

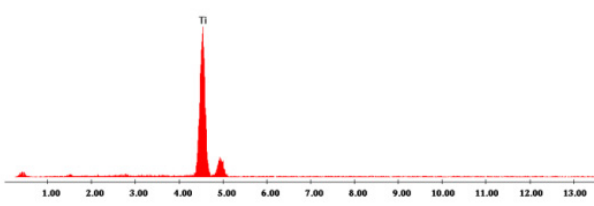

T-4

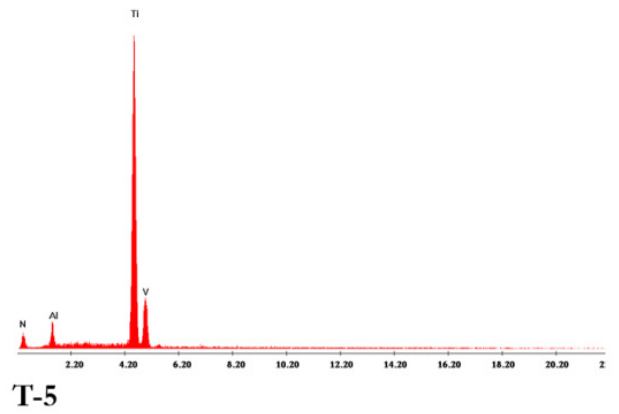

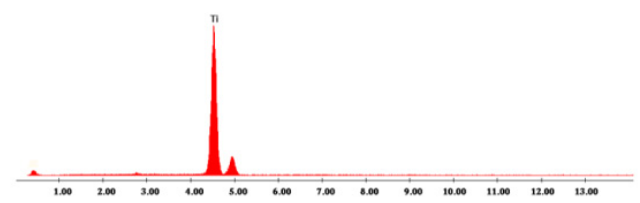

MA-4

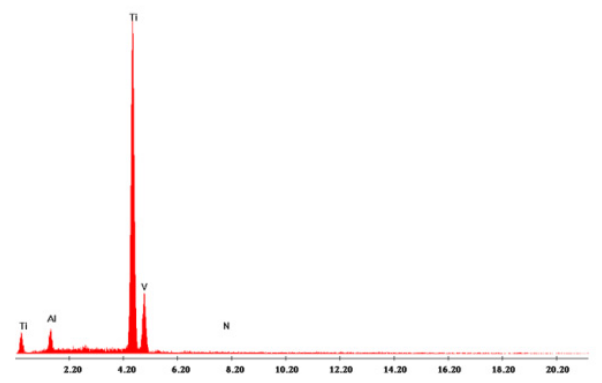

MA-5

Figure 2. Energy Dispersive X-ray Spectrometry (SEM/EDS) analysis of the turned grade 4 (T-4), turned grade 5 (T-5), mildly acid-etched grade 4 (MA-4), and mildly acid-etched grade 5 (MA-5) Ti surfaces.

\subsubsection{D Surface Profiling}

Table 1 reports the values of Sa, Sds and Sdr for the tested surfaces after the application of an $80 \mu \mathrm{m}$ Gauss' filter. 
Table 1. Surface parameters of turned grade 4 (T-4), turned grade 5 (T-5), mildly acid-etched grade 4 (MA-4), and mildly acid-etched grade 5 (MA-5) Ti surfaces. Data are expressed as mean \pm standard deviation. $\mathrm{N}=9$ /group; ${ }^{*}=p<0.05$ vs. T-4; $\mathrm{a}=p<0.05$ vs. T-5. Sa: absolute deviation of the surface; Sds: density of summits of the surface; Sdr: developed surface area ratio.

\begin{tabular}{cccc}
\hline Sample Code & Sa $(\boldsymbol{\mu m})$ & Sds $\left(\boldsymbol{\mu \mathbf { m } ^ { - 2 } )}\right.$ & Sdr $(\mathbf{\%})$ \\
\hline T-4 & $0.126 \pm 0.04$ & $0.243 \pm 0.01$ & $10.47 \pm 7.50$ \\
T-5 & $0.134 \pm 0.02$ & $0.249 \pm 0.01$ & $11.16 \pm 7.12$ \\
MA-4 & $0.225 \pm 0.02 *$ & $0.218 \pm 0.01$ & $12.43 \pm 4.51$ \\
MA-5 & $0.212 \pm 0.02$ a & $0.205 \pm 0.05$ & $8.31 \pm 7.65$ \\
\hline
\end{tabular}

Within the investigated testing conditions and for the adopted equipment, MA surfaces showed significantly higher Sa values compared to $\mathrm{T}$ samples. On the contrary, no significant difference of Sds or Sdr was detected between MA and T surfaces, or between grade 4 and grade 5 surfaces.

\subsection{Bacterial Adhesion In Vitro}

Isolated colonies of $S$. sanguinis on T surfaces, and areas covered by an almost continuous bacterial layer on MA surfaces, were visible $6 \mathrm{~h}$ after in vitro bacteria inoculation at the ESEM analysis (Figure 3).

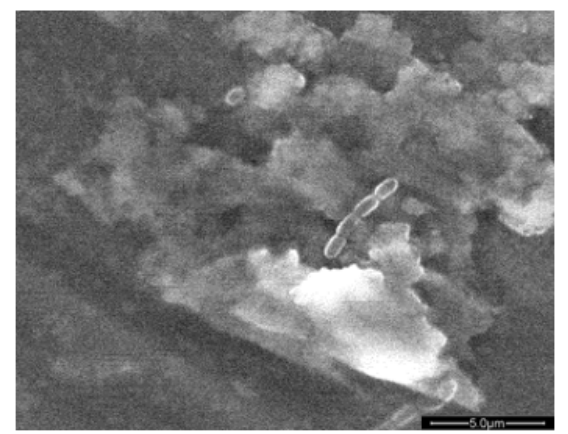

T-4

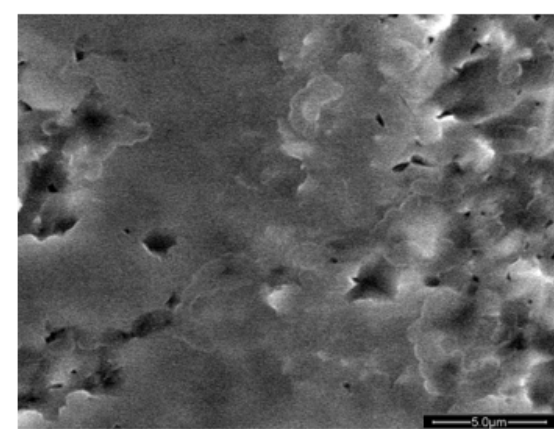

MA-4

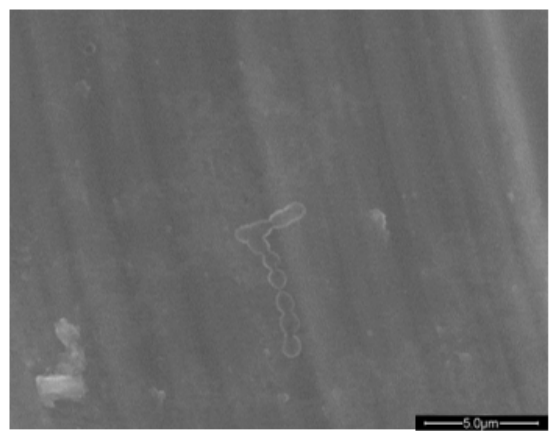

T-5

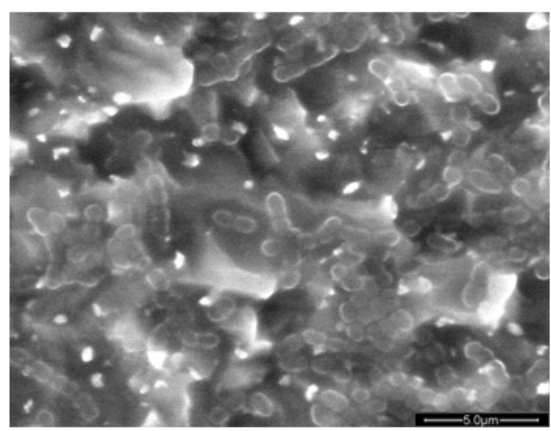

MA-5

Figure 3. Environmental scanning electron microscope (ESEM) images of the turned grade 4 (T-4), turned grade 5 (T-5), mildly acid-etched grade 4 (MA-4), and mildly acid-etched grade 5 (MA-5) Ti surfaces $6 \mathrm{~h}$ after inoculation of a suspension of S. sanguinis. Magnification 5000×.

Figure 4 shows the number of attached bacteria on each sample, quantified by CFU counting, 2 and $6 \mathrm{~h}$ after bacteria inoculation. S. sanguinis showed a significantly higher adhesion on the MA surfaces compared to the respective T surfaces, both at 2 and $6 \mathrm{~h}$ after plating. No difference was found between grade 4 and grade $5 \mathrm{Ti}$ surfaces. 


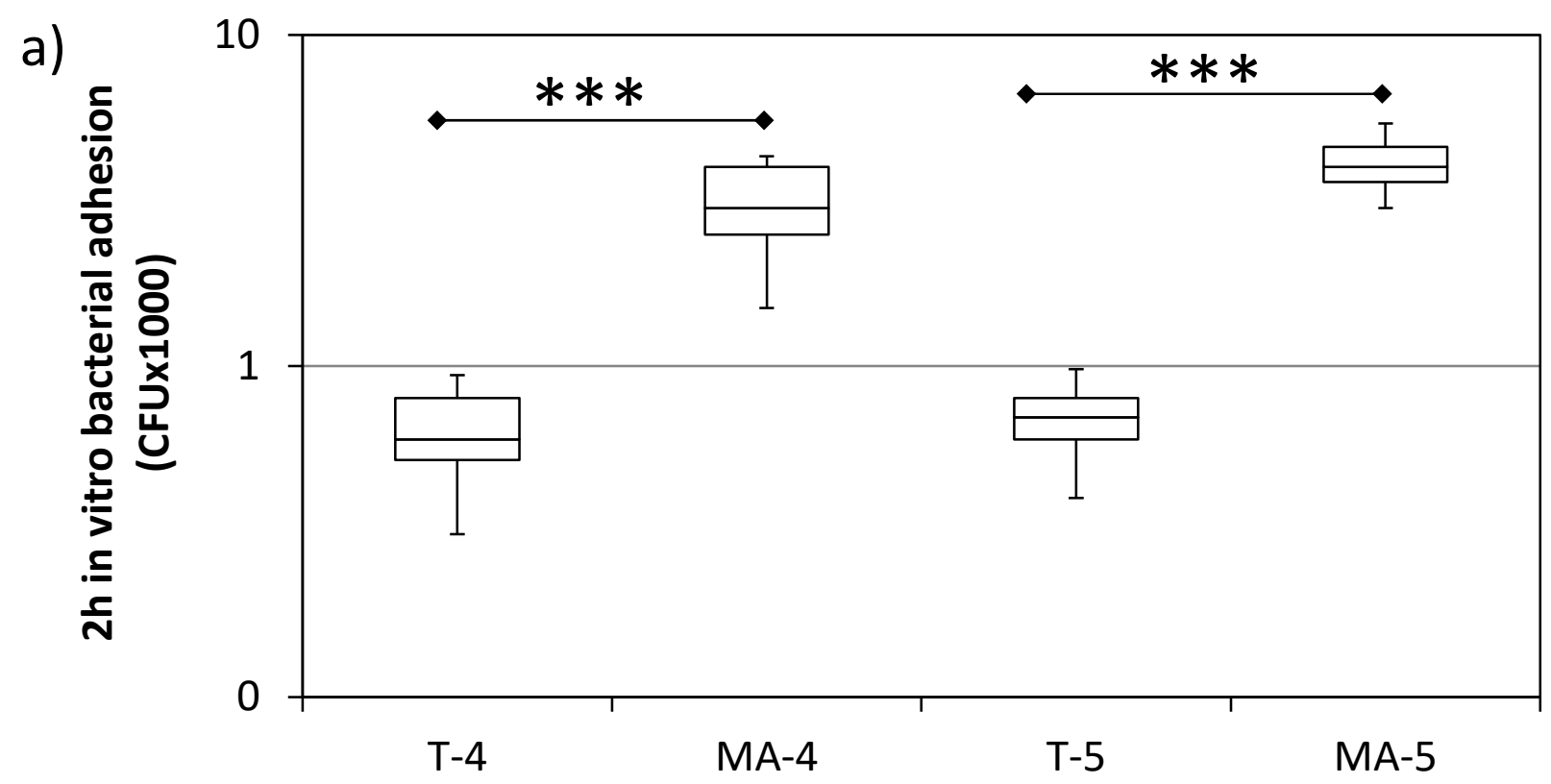

b)

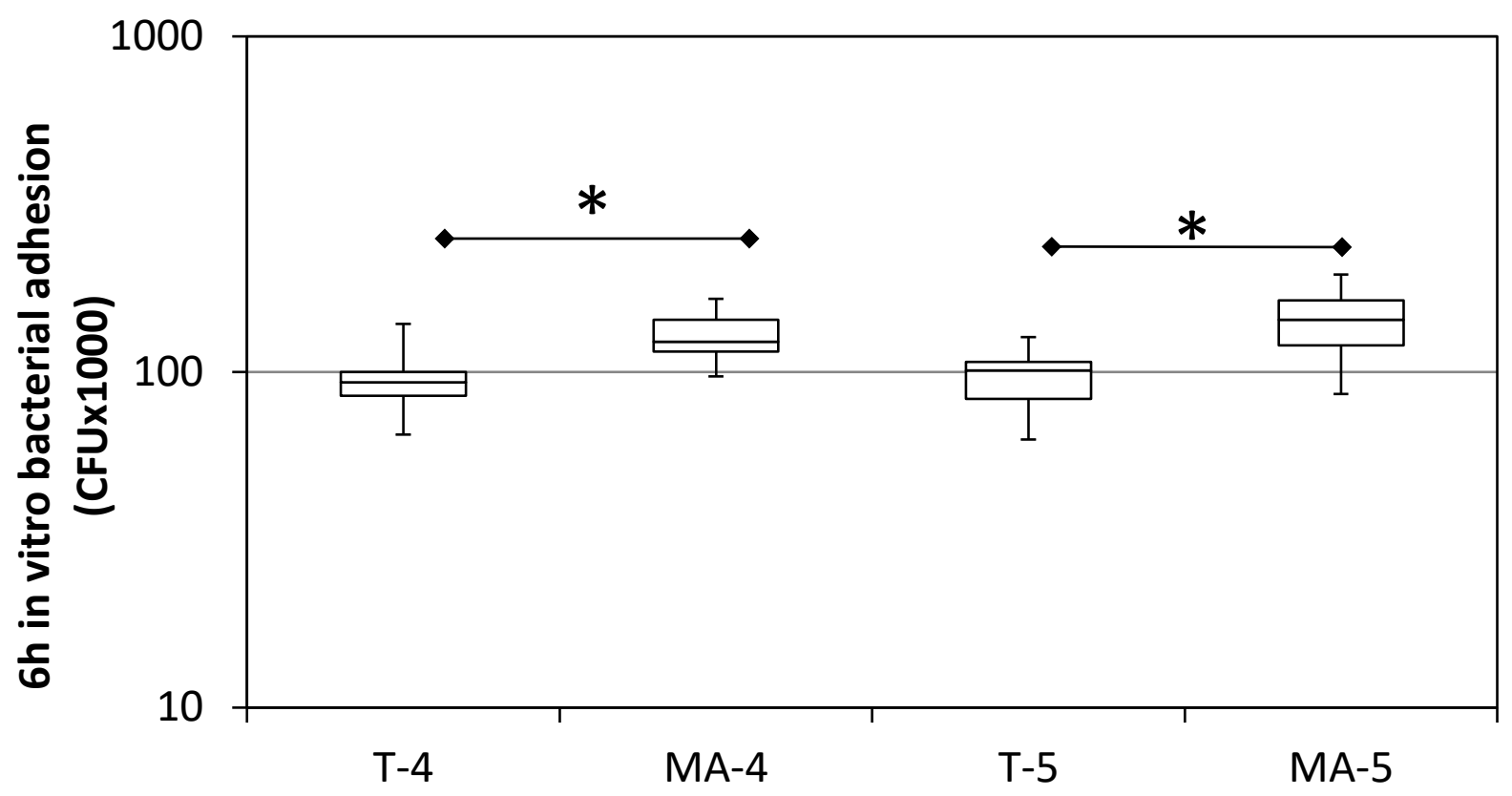

Figure 4. In vitro bacterial adhesion (S. sanguinis) on turned grade 4 (T-4), turned grade 5 (T-5), mildly acid-etched grade 4 (MA-4), and mildly acid-etched grade 5 (MA-5) Ti surfaces at $2 \mathrm{~h}(\mathbf{a})$ and $6 \mathrm{~h}$ (b) after inoculation. Data are expressed as counts of colony forming units (CFU) on a logarithmic scale (median, interquartile range, minimum $/$ maximum). $\mathrm{N}=9$. ${ }^{*}=p<0.05 ;{ }^{* * *}=p<0.001$.

\subsection{Ex Vivo Bacterial Adhesion}

After a $12 \mathrm{~h}$-permanence of Ti samples in the oral cavity, significantly higher values of CFU count for the MA surfaces (about 10-fold) compared with the respective T surfaces were found (Figure 5). Significantly higher values were also detected for grade $5 \mathrm{~T}$ and grade $5 \mathrm{MA}$ Ti samples compared with the respective grade 4 surfaces (about 3.5 and 2 times higher, respectively). 


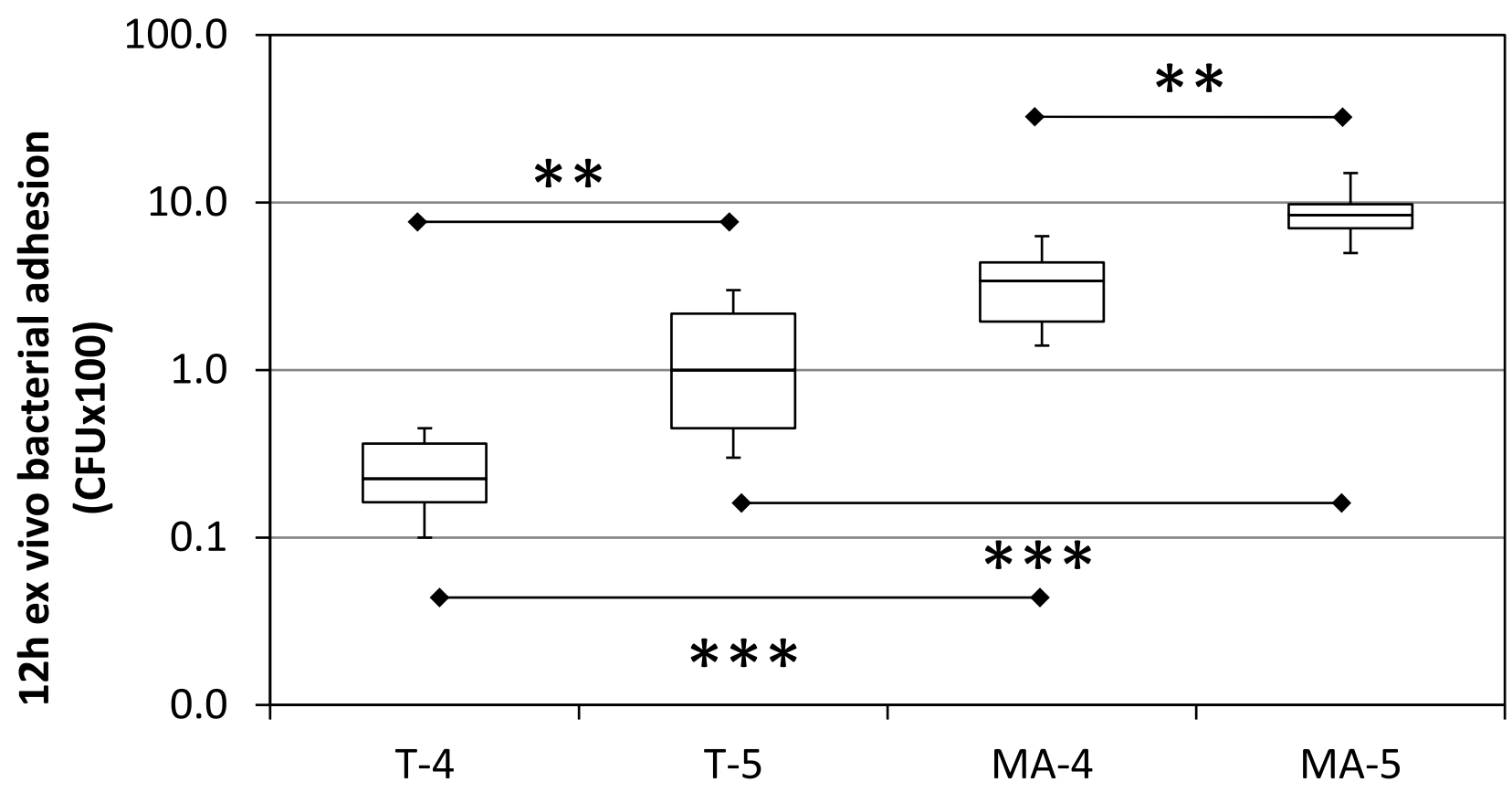

Figure 5. Bacterial adhesion on turned (T), mildly acid-etched (MA), grade 4 and grade $5 \mathrm{Ti}$ implant surfaces after $12 \mathrm{~h}$ ex vivo staying in the mouth. Data are expressed as counts of Colony Forming Units (CFU) on a logarithmic scale (median, interquartile range, minimum/maximum). $\mathrm{N}=12 .{ }^{* *}=p<0.01 ;{ }^{* * *} p<0.001$.

\section{Discussion}

In this present study, we have demonstrated not only topographic features of Ti implant surfaces, but also how their chemical composition may affect their microbiological properties.

The topographic characterization of tested implant surfaces was carried out using multiple analysis parameters and appropriate filtering to obtain an exhaustive description of their texture and reduce the errors due to form and waviness, as suggested by Wennerberg and Albrektsson (2000) [16]. Following the classification of implant surfaces proposed by these authors, both T and MA tested surfaces can be considered "smooth" (Sa $<0.5 \mu \mathrm{m})$. Of note, most of the implants on the market, including those roughened by acid-etching techniques, have a minimally rough $(\mathrm{Sa} 0.5-1.0 \mu \mathrm{m})$ or moderately rough (Sa 1.0-2.0 $\mu \mathrm{m})$ surface [17]. Nevertheless, MA surfaces showed significantly higher Sa values than T ones, whereas the chemical composition of tested specimens (grade 4 vs. grade $5 \mathrm{Ti}$ ), did not seem to affect surface topography. The low roughness values found for MA samples could be due to the mild organic acids (e.g., oxalic and maleic acid) used for their treatment, resulting in a surface texture characterized by a sequence of multiple pits and ridges less pronounced than those of strong acids conventionally employed (e.g., phosphoric acid, hydrochloric acid) (see Figure 1).

The use of in vitro models in studies on microbial adhesion allows reproducibility, cost-effectiveness, and control of possible influencing variables. On the other hand, the translation of in vitro results to clinical reality is more difficult. In this sense, ex vivo experimental models offer the chance to investigate these phenomena in conditions closer to the clinical ones, with a heterogenous biofilm, composed of a variety of symbiotic microorganisms, with the presence of an acquired pellicle of salivary origin, and with the detaching forces impressed by the salivary flow and the functional action of peri-oral structures [18]. The microorganism chosen for the in vitro part of the present study was the S. sanguinis, a common resident of the oral cavity, due to its role of initial colonizer of the human oral biofilm [19]. The sonication protocol for bacteria detachment was preferred among other available techniques (e.g., scraping) due to its high efficiency and recovery rate, as previously demonstrated [20]. Incubation times of 2 and $6 \mathrm{~h}$ were chosen in the present study to investigate in vitro the initial phase of bacterial adhesion on implant 
surfaces. On the other hand, a longer time (12-24 h) is needed using ex vivo study models to obtain a structured biofilm upon Ti surfaces $[18,21]$.

We found in vitro a significantly higher CFU count of S. sanguinis on MA compared to $\mathrm{T}$ samples after 2 and $6 \mathrm{~h}$ of incubation. The absolute values of all groups tend to increase over time, whereas the inter-group difference tends to markedly decrease from 2 to $6 \mathrm{~h}$, although maintaining the statistical significance. In a longer period of observation, in the ex vivo experimentation, such a difference was even more pronounced than in vitro, although CFU absolute values ex vivo were lower than those found in vitro. CFU values were found about 10-fold higher on MA than T surfaces and, furthermore, 2 to 3.5-fold higher on grade 5 than grade 4 surfaces. It must be underlined that a direct comparison between in vitro and ex vivo results at the different time points cannot be made due to the significantly different experimental conditions applied, and that several environmental parameters potentially affecting bacterial adhesion (e.g., $\mathrm{pH}$, composition, etc.) may have also played a role in determining such discrepancies between these two experimental approaches. A possible explanation, for instance, can be probably found in, but not limited to, the sheltering effect that rough surfaces offer ex vivo to the microbes against the shear removal forces of the oral environment [18].

Only a few studies in the literature have investigated bacterial adhesion on Ti surfaces of different grades, with contrasting results [14,15]. In the study of Barão et al. (2014) [14] commercially pure $\mathrm{Ti}(\mathrm{cp}-\mathrm{Ti})$ and grade 5 (Ti-6Al-4V alloy) disks were inoculated with $P$. gingivalis in artificial saliva at different $\mathrm{pHs}$, and bacterial adhesion evaluated after $6 \mathrm{~h}$. Different from our results, significantly more bacterial adhesion to the cp-Ti disks than to the Ti-6Al-4V alloy disks was shown. In the study of Di Giulio et al. (2016) [15], grade 4 and grade 5 Ti disks were incubated with P. gingivalis for $48 \mathrm{~h}$ and the biofilm biomass spectrophotometrically quantified. In this case, as for our study, a significantly higher biofilm formation was detected on grade 5 than grade 4 surfaces when machined and lasertreated specimens were tested, whereas no effect of Ti composition on biofilm biomass formation for sandblasted surfaces was found. Such discrepancies and similarities among those studies and the present one must be interpreted with caution due to the different design, bacteria and materials used. The use of an ex vivo model to reproduce conditions closer to the clinical reality, for instance, represents a significant difference and novelty of the present study compared with the above-mentioned ones.

Surface properties, in particular surface roughness, of implant surfaces are directly correlated with bacterial adhesion, as demonstrated in both in vitro and ex vivo experimental models in the present study, as well as in the existing literature. John et al. (2015) [22] measured initial biofilm growing ( $48 \mathrm{~h}$ after inoculation) on four Ti surfaces different for texture or chemical composition. Machined surfaces were reported to favor a slower biofilm formation and maturation than the roughened surfaces. The higher tendency of rough surfaces to accumulate and retain more mature plaque can be found in their ability to provide locations for initial adhesion in which bacteria are sheltered against shear forces [6]. Although a number of studies have tried to correlate surface roughness parameters with bacterial adhesion [23,24], this association is still far from being fully determined. The profile of areal average roughness ( $\mathrm{Ra}$ or $\mathrm{Sa}$ ) remains the most extensively investigated roughness parameter for implant surfaces, and a strong correlation between bacterial adhesion with the Sa values of differently micro-roughened surfaces has been shown $[6,18,23,24]$. Bollen et al., in their studies, have proposed a threshold value of roughness $(\mathrm{Sa}=0.2 \mu \mathrm{m})$ below which we cannot expect further reduction of bacterial adhesion $[23,24]$. Our findings, however, cannot confirm this aspect [25], because we found a significantly lower biofilm formation on T surfaces (Sa about $0.13 \mu \mathrm{m}$ ) compared to MA ones.

The existing positive correlation between surface roughness and bacterial adhesion has a direct influence on the producing process and design of dental implants and related components; transmucosal implant parts and components are conventionally mirror-like polished, whereas in cases where the intraosseous rough surface is exposed for some reason to the oral environment, bacterial adhesion and, possibly, peri-implant disease 
(mucositis and peri-implantitis) could be promoted. However, the correlation between implant surface characteristics and the incidence of peri-implant disease is still far from being clearly defined. Available literature data are very heterogeneous, and it is difficult to extrapolate in vitro and in vivo findings to the clinical field, also due to the multifactorial nature of the disease [26-29]. In a 2017 meta-analysis [30] performed on 87 studies with at least five years of follow-up, the authors concluded that peri-implant bone loss around minimally rough implant systems is statistically less significant in comparison to the moderately rough and rough implant systems, which, in turn, show comparable results to each other. Furthermore, in history of periodontitis patients, rough surfaces have been suggested to be more prone to developing marginal bone loss and peri-implantitis than nonperiodontal patients, whereas such differences were not shown for machined surfaces [31]. Additionally, the response to common therapeutic approaches to peri-implantitis has been shown to be possibly affected by surface characteristics. The treatment of peri-implantitis cannot still be considered fully predictable at the present [32]. Different non-surgical and surgical approaches (e.g., mechanical instrumentation, air-polishing, local and systemic antimicrobials, laser photodynamic therapy) have been proposed for implant surface decontamination [33-35], with conflicting results, and surface properties could have a role in affecting the success of such treatments and the risk of recurrence of the disease [36,37].

\section{Conclusions}

In conclusion, our findings indicate that:

- Bacterial adhesion, particularly when tested ex vivo, was shown to be sensitive to both Ti surface topography and composition. MA surfaces were shown to be more attractive for bacteria than T surfaces, although having similar roughness values. Even slight differences in the surface topography of tested surfaces were found to have a significant effect on the bacterial adhesion.

- Such findings could have possible implications on peri-implant tissue health maintenance. Turned surfaces, especially grade 4 Ti ones, could be more advantageously used for transmucosal implant parts and components, whereas mildly acid-etched surfaces, especially grade 5 Ti ones, should be kept far from the oral environment, remaining confined at the endosseous level, being more prone to bacterial colonization.

- Further studies are needed to investigate the effect of chemical and topographic characteristics of implant surfaces on bacterial adhesion and peri-implant tissue health. In order to obtain a more accurate topographic surface characterization, the use of high resolution equipment, 3D methods, multiple parameters and filtering procedures is recommended, as well as the use of ex vivo experimental models to increase the clinical relevance of the obtained results.

Author Contributions: M.A., G.C., L.G., L.S.: conceptualization; C.L.: topographical analysis; R.G. and L.N.: data analysis; M.A., G.C., R.G., L.G., C.L., L.N., L.S: manuscript writing; M.A. and L.G.: supervision; all the authors revised and edited the manuscript. All authors have read and agreed to the published version of the manuscript.

Funding: This research received no external funding.

Institutional Review Board Statement: The study was conducted according to the guidelines of the Declaration of Helsinki, and approved by the Ethics Committee of the University of Campania "Luigi Vanvitelli" (\#602, 22 April 2015).

Informed Consent Statement: Informed consent was obtained from all subjects involved in the study.

Acknowledgments: We thank Leader Italia s.r.l. (Milano, Italy) for providing the Ti samples used in the present study and Arcangelo Pignalosa (dental tecnician at the University of Campania "Luigi Vanvitelli", Naples, Italy) for the production of the acrylic templates used for the ex vivo tests.

Conflicts of Interest: The authors declare no conflict of interest. 


\section{References}

1. Howe, M.-S.; Keys, W.; Richards, D. Long-term (10-year) dental implant survival: A systematic review and sensitivity metaanalysis. J. Dent. 2019, 84, 9-21. [CrossRef]

2. Albrektsson, T.; Brånemark, P.-I.; Hansson, H.-A.; Lindström, J. Osseointegrated Titanium Implants:Requirements for Ensuring a Long-Lasting, Direct Bone-to-Implant Anchorage in Man. Acta Orthop. Scand. 1981, 52, 155-170. [CrossRef]

3. Quirynen, M.; De Soete, M.; Van Steenberghe, D. Infectious risks for oral implants: A review of the literature. Clin. Oral Implant Res. 2002, 13, 1-19. [CrossRef] [PubMed]

4. Wennerberg, A.; Albrektsson, T. Effects of titanium surface topography on bone integration: A systematic review. Clin. Oral Implant Res. 2009, 20, 172-184. [CrossRef]

5. Annunziata, M.; Guida, L. The Effect of Titanium Surface Modifications on Dental Implant Osseointegration. Biomater. Oral Craniomaxillofacial Appl. 2015, 17, 62-77.

6. Teughels, W.; Van Assche, N.; Sliepen, I.; Quirynen, M. Effect of material characteristics and/or surface topography on biofilm development. Clin. Oral Implant Res. 2006, 17, 68-81. [CrossRef] [PubMed]

7. Subramani, K.; Jung, R.E.; Molenberg, A.; Hämmerle, C.H.F. Biofilm on dental implants: A review of the literature. Int. J. Oral Maxillofac. Implant 2009, 24, 616-626. [CrossRef]

8. Renvert, S.; Quirynen, M. Risk indicators for peri-implantitis. A narrative review. Clin. Oral Implant Res. 2015, 26, 15-44. [CrossRef]

9. Barone, A.; Orlando, B.; Cingano, L.; Marconcini, S.; Derchi, G.; Covani, U. A randomized clinical trial to evaluate and compare implants placed in augmented versus non-augmented extraction sockets: 3-year results. J. Periodontol. 2012, 83, 836-846. [CrossRef]

10. Schwarz, F.; Derks, J.; Monje, A. Peri-implantitis. J. Periodontol. 2018, 89, S267-S290. [CrossRef]

11. McCracken, M. Dental Implant Materials: Commercially Pure Titanium and Titanium Alloys. J. Prosthodont. 1999, 8, 40-43. [CrossRef] [PubMed]

12. Liu, X.; Chen, S.; Tsoi, J.K.; Matinlinna, J.P. Binary titanium alloys as dental implant materials-A review. Regen. Biomater. 2017, 4, 315-323. [CrossRef] [PubMed]

13. Elias, C.N.; Fernandes, D.; de Souza, F.M.; Monteiro, E.D.S.; de Biasi, R.S. Mechanical and clinical properties of titanium and titanium-based alloys (Ti G2, Ti G4 cold worked nanostructured and Ti G5) for biomedical applications. J. Mater. Res. Technol. 2019, 8, 1060-1069. [CrossRef]

14. Barão, V.A.R.; Yoon, C.J.; Mathew, M.T. Attachment of Porphyromonas gingivalis to corroded commercially pure titanium and titanium-aluminum-vanadium alloy. J. Periodontol. 2014, 85, 1275-1282. [CrossRef]

15. Di Giulio, M.; Traini, T.; Sinjari, B. Porphyromonas gingivalis biofilm formation in different titanium surfaces, an in vitro study. Clin. Oral. Implants Res. 2016, 27, 918-925. [CrossRef]

16. Wennerberg, A.; Albrektsson, T. Suggested guidelines for the topographic evaluation of implant surfaces. Int. J. Oral Maxillofac. Implant 2000, 15, 331-344.

17. De Bruyn, H.; Christiaens, V.; Doornewaard, R. Implant surface roughness and patient factors on long-term peri-implant bone loss. Periodontol. 2000 2017, 73, 218-227. [CrossRef]

18. Bürgers, R.; Gerlach, T.; Hahnel, S. In vivo and in vitro biofilm formation on two different titanium implant surfaces. Clin. Oral Implants Res. 2010, 21, 156-164. [CrossRef]

19. Abranches, J.; Zeng, L.; Kajfasz, J.K.; Palmer, S.R.; Chakraborty, B.; Wen, Z.T.; Richards, V.; Brady, L.J.; Lemos, J. Biology of Oral Streptococci. Microbiol. Spectr. 2018, 6. [CrossRef]

20. Bjerkan, G.; Witsø, E.; Bergh, K. Sonication is superior to scraping for retrieval of bacteria in biofilm on titanium and steel surfaces in vitro. Acta Orthop. 2009, 80, 245-250. [CrossRef]

21. Größner-Schreiber, B.; Griepentrog, M.; Haustein, I.; Müller, W.-D.; Briedigkeit, H.; Göbel, U.B.; Lange, K.-P. Plaque formation on surface modified dental implants. Clin. Oral Implant Res. 2001, 12, 543-551. [CrossRef] [PubMed]

22. John, G.; Becker, J.; Schwarz, F. Modified Implant Surface with Slower and Less Initial Biofilm Formation. Clin. Implant Dent. Relat. Res. 2013, 17, 461-468. [CrossRef] [PubMed]

23. Bollen, C.M.L.; Papaioanno, W.; Van Eldere, J.; Schepers, E.; Quirynen, M.; Van Steenberghe, D. The influence of abutment surface roughness on plaque accumulation and peri-implant mucositis. Clin. Oral Implant Res. 1996, 7, 201-211. [CrossRef] [PubMed]

24. Bollenl, C.M.; Lambrechts, P.; Quirynen, M. Comparison of surface roughness of oral hard materials to the threshold surface roughness for bacterial plaque retention: A review of the literature. Dent. Mater. 1997, 13, 258-269. [CrossRef]

25. Annunziata, M.; Rizzo, A.; Leone, C.; Mangano, C.; Mazzola, N.; Nastri, L.; Papale, F.; Rullo, F.; Guida, L. Bacterial adhesion to direct laser metal formed and mildly acid etched implant surfaces. Surf. Coat. Technol. 2017, 328, 390-397. [CrossRef]

26. Canullo, L.; Annunziata, M.; Pesce, P.; Tommasato, G.; Nastri, L.; Guida, L. Influence of abutment material and modifications on peri-implant soft-tissue attachment: A systematic review and meta-analysis of histological animal studies. J. Prosthet. Dent. 2021, 125, 426-436. [CrossRef]

27. Schwarz, F.; Mihatovic, I.; Golubovic, V.; Eick, S.; Iglhaut, T.; Becker, J. Experimental peri-implant mucositis at different implant surfaces. J. Clin. Periodontol. 2014, 41, 513-520. [CrossRef]

28. Quirynen, M.; Van Assche, N. RCT comparing minimally with moderately rough implants. Part 2: Microbial observations. Clin. Oral Implant Res. 2011, 23, 625-634. [CrossRef] [PubMed] 
29. Renvert, S.; Lindahl, C.; Persson, G.R. The incidence of peri-implantitis for two different implant systems over a period of thirteen years. J. Clin. Periodontol. 2012, 39, 1191-1197. [CrossRef] [PubMed]

30. Doornewaard, R.; Christiaens, V.; De Bruyn, H. Long-Term Effect of Surface Roughness and Patients' Factors on Crestal Bone Loss at Dental Implants. A Systematic Review and Meta-Analysis. Clin. Implant Dent. Relat. Res. 2017, 19, 372-399. [CrossRef]

31. Lin, C.-Y.; Chen, Z.; Pan, W.-L.; Wang, H.-L. Is History of Periodontal Disease Still a Negative Risk Indicator for Peri-implant Health under Supportive Post-Implant Treatment Coverage? A Systematic Review and Meta-analysis. Int. J. Oral Maxillofac. Implant 2020, 35, 52-62. [CrossRef]

32. Figuero, E.; Graziani, F.; Sanz, I.; Herrera, D.; Sanz, M. Management of peri-implant mucositis and peri-implantitis. Periodontol. 2000 2014, 66, 255-273. [CrossRef]

33. Schwarz, F.; Schmucker, A.; Becker, J.C. Efficacy of alternative or adjunctive measures to conventional treatment of peri-implant mucositis and peri-implantitis: A systematic review and meta-analysis. Int. J. Implant Dent. 2015, 1, 1-34. [CrossRef] [PubMed]

34. Barrak, I.; Baráth, Z.; Tián, T.; Venkei, A.; Gajdács, M.; Urbán, E.; Stájer, A. Effects of different decontaminating solutions used for the treatment of peri-implantitis on the growth of Porphyromonas gingivalis-an in vitro study. Acta Microbiol. Immunol. Hung. 2021, 68, 40-47. [CrossRef] [PubMed]

35. Stájer, A.; Kajári, S.; Gajdács, M.; Musah-Eroje, A.; Baráth, Z. Utility of Photodynamic Therapy in Dentistry: Current Concepts. Dent. J. 2020, 8, 43. [CrossRef] [PubMed]

36. Carcuac, O.; Derks, J.; Charalampakis, G. Adjunctive Systemic and Local Antimicrobial Therapy in the Surgical Treatment of Peri-implantitis: A Randomized Controlled Clinical Trial. J. Dent. Res. 2016, 95, 50-57. [CrossRef] [PubMed]

37. Carcuac, O.; Derks, J.; Abrahamsson, I.; Wennström, J.L.; Berglundh, T. Risk for recurrence of disease following surgical therapy of peri-implantitis-A prospective longitudinal study. Clin. Oral Implant Res. 2020, 31, 1072-1077. [CrossRef] 